\section{Causes of Newborn Asphyxia in Pudong Zong Bai Zhenggang}

Pudong Gongli Hospital, Shanghai, PEOPLE'S REPUBLIC OF CHINA

This study is an analysis of 69 cases of asphyxia of the newborn from 1994 to 1999. The findings indicate that there are a variety of factors that contribute to asphyxia of the newborn. Newborn asphyxia results mainly from the circulatory interruption of inner uterus, umbilical cord around the neck of the baby, and/or pollution and aspiration of amniotic fluid. Besides the maternal factors, newborn factors include abnormal fetal position and placental factors that contribute to the newborn asphyxia. CT tests of some of the newborn's brains indicates that the incidence rate of hypoxic-ischemic encephalopathy (HIE) approaches $82.6 \%$, and for intracranial hemorrhage, up to $73.9 \%$. Thus, it is quite necessary to prevent newborn asphyxia, reduce the degree of asphyxia, and avoid the occurrence of sequellae, by virtue of the following: (1) monitoring the fetus inside the uterus early and prevent problems with the inner uterus; (2) having an immediate abdominal delivery in the necessary situations; (3) taking a B ultrasonic inspection, so as to make certain of the chances that the umbilical cord is not abnormal before childbirth; and (4) taking a $\mathrm{CT}$ test for the brain, under good conditions.

One particular concern is that immigrating workers are reluctant to see a medical professional before childbirth, and/or are willing to have a delivery at home due to financial hardship, or refuse to come to the hospital until they are in labor and are about to deliver the baby. This lack of medical care contributes to the rise in the newborn asphyxia and its sequellae. Thus, informing the public must be emphasized

Key words: asphyxia; causes; diagnosis; neonatal; newborn; obstetrics; prevention

Prebosp Disast Med 2001;16(2):s16.

\section{Anaesthetist Nurse on a Humanitarian Mission Isabelle Ballanger, Anaesthetist Nurse Lyon, FRANCE}

The conflict in Burundi between the Tutsi power and the majority Hutu, which started in 1963, has escalated since 1993. Many factions have appeared, and a peace agreement has not been achieved. As the battles have become more intense over the recent years, the number of victims who have been entrusted to an already precarious health system has increased, justifying the need for medical help from Médecins sans Frontières (France). The daily medical needs (obstetrics, visceral, trauma) are augmented by the irregular, and, at times, massive influx of injuries as a result of the effects of constant war. The humanitarian assistance takes the shape of human resources (a team surgeon, an anaesthetist nurse or doctor, a field coordinator to negotiate with the provincial authorities, a coordinator for the general daily management), logistics (medicine, standard kits that include a sterilizer, a box of surgical instruments, an oxygen extractor, manual ventilation material, pulse oximeter, etc.) and medical know-how from 30 years of experience in difficult conditions. The medical team demonstrates its professional competencies and qualities of human relations by taking charge of any surgical casualties, adjusting to a minimal technical environment, partnership (work and formation) with the local health service, communication with the nursed populations, development of a team spirit (between expatriates who aren't used to working together) and the constant control of security problems. The expatriate anaesthetist nurse or doctor is the only representative of this specialty, and controls the anaesthesia (general anaesthesia with or without intubation, spinal analgesia, regional intravenous anaesthesia, nerve block anaesthesia), postoperative care (hydro-electrolytic infusion, analgesia therapy, antibiotic therapy, antitetanus vaccination, medicine supply, dressing change, etc, control and management of anaesthetic and surgical equipment and sterilisation, involvement in the care and the hospital hygiene with local hospital staff, and monitoring this activity by weekly and monthly reports. Tense security issues forced the team to adjourn its activity on 22 May 2000. No governmental authority has yet permitted its reinstatement, in spite of very important medical civilian and military needs. The complex situation of this country in wartime shows the limits of the aide programs in crisis situations.

Key words: anaethetists; expatriates; Hutu; logistics; needs; security; supplies; team; Tutsi; war

Prebosp Disast Med 2001;16(2):s16.

\section{Systematic Radiologic Checking of Gastric Tubes in ICU and Emergency Services: Preliminary Results S. Beague; P. Mielcarek; T. Onimus; F. Saulnier; S. Nseir; A. Durocher \\ Calmette ICU, CHRU Lille, FRANCE}

Introduction: There is a professional agreement concerning the radiological checking of the position of gastric tubes (GT) after their installation in the field of continual enteral feeding for adults, that it is at hospital or at home. ${ }^{1}$ This research evaluated the relevance of this recommendation in emergencies and also sought predictive factors of difficulty encountered with the installation of GTs or anomalies of localization of the GT after its installation.

Methods: It is a prospective study. All patients admitted in our service and having profited from the installation of a GT, prehospital or not, were included. The data collected are: (1) demographic data of the patient, (2) admission data (antecedents, principal diagnosis, CGS, needs of intubation, tracheotomy or sedation), (3) mode of installation of the GT, (4) its type, and (5) existence of difficulties during this installation. Checking of the position of GT is achieved by injection of air and radiological control. The statistical tests used were Student's t-test for quantitative information and a chi-square test for qualitative data. A value of $p<0.05$ was considered statistically significant.

Results: 81 installations of GT were studied. The median age of patients is 58 years (19-94). 53\% of patients were men, $32.5 \%$ of patients were sedated. Neurological or gas- 